scatter." It is obvious that the smallest opacity or haze will be clearly visible.

When the beam is thrown into the eye obliquely it illuminates a prismatic section of the cornea and of the lens. If the ribbon of light is made very thin this is in effect an optical section. The section of the cornea magnified twenty-five times is two centimetres thick and enables opacities and alterations to be accurately localized. The anterior chamber remains dark unless the aqueous contains particles which can be clearly seen if the light and microscope are accurately focused upon them; they will be noted to move with a streaming motion, caused by the heat eddy in the anterior chamber due to the difference in temperature between the iris and cornea. The motion is accelerated by the heat of the beam of light. Whereas the presence of cellular elements is the first sign of irido-crclitis the detection of this movement may be of great clinical significance, and may give timely warning of an impending attack of sympathetic ophthalmitis.

The lens seen in optical section shows a series of lamellae, areas of varying refractive index. Vogt divides them into the capsule, the cortex, the senile nucleus, and the embryonic nucleus. The latter corresponds to the lens in later foetal life. The ability to localize opacities in one or other of these layers has a prognostic significance, for a cataract confined to the cortex cannot be congenital, this part of the lens developing after foetal life.

The vitreous shows a framework structure which varies in different individuals; in fact, in many it appears to be optically homogeneous till examined with a slit-lamp furnished with a micro-arc lamp. It is interesting to note that the slit-lamp has shown that persistence of the hyaloid artery in rudimentary form is almost constant and is not the rarity that was supposed.

The clinical value of the slit-lamp is immense, fund sirce I have used one at the Coventry Hospital I feel lost without it. It is obvious that an instrument which enables us to see the blood circulating in the corneal vessels, that shows up cells in the aqueous, and by means of which we can make an accurate localization of objects in the transparent media cannot be neglected much longer. The slit-lamp has for the past three years found a place in every Continental clinic, and at Zürich, under Professor Vogt, it is used for nearly every patient. It turns what at best is good guessing to certainty, and opens the way for intensive research.

The address was illustrated by epidiascope projection from Vogt's Atlas of Slit-Lamp Microscopy, and from Koeppe's Microscopy of the Liring Eye.

\section{THE ACTION OF PHYSOSTIGMINE AND PITUITRIN :}

The ACtion of these Drugs, Alone AND Combined, upon THE IsOLATED HUMAN VERMIFORM APPENDIX; THE ADVANTAGES OF THEIR COMBINED USE IN Post-operative Ileus.

BY

D. G. T. KERR CROSS, B.A., M.B., B.Ch.Oxon., Radcliffe Traveluing Fellow, 1922.

(From the Laboratories of the Pharmacological Department, Oxford University.)

Sisce the early days of abdominal surgery post-operative atony of the intestines or ileus has been a menace to recovery. The signs and symptoms are closely akin to those of acute mechanical intestinal obstruction. The distinction was clearly drawn by Nothnagel, ${ }^{1}$ who divided ileus into two classes-

1. Mechanical, from occlusion of the bowel-(a) by obstruction from within; (b) spasm or constricting disease in its own substance; $(c)$ pressure from without, adhesions, tumours, etc. ; $(d)$ twists, kinks, invaginations, etc.

2. Dynamic ileus, in which there is the syndrome of occlusion, with no closure of the lumen, this occurring when the intestine becomes paralysed.

With the first group this paper is not concerned; there is fortunately no more appreciated fact in modern surgery than that immediate laparotomy is required.

In regard to dynamic ileus Cannon and Murphy ${ }^{2}$ have concluded that this condition is caused (1) by inhibitory impulses via the splanchnic nerves; (2) by injury o." depression of Auerbach's myenteric plexus: (3) by trauma to the muscle itself. They proved this by the radiographic examination of opaque meals given to cats $(a)$ after ether anaesthesia, (b) after ether anaesthesia during which the testicles were crushed, $(c)$ as in $(b)$, the splanchnic nerves having been cut some days before. After $(a)$ the meal passed normally; after $(b)$ the meal passed hardly at all because the shocked animal sent inhibitory messages along its splanchnic nerves; after $(c)$ the meal passed almost normally because messages could not pass along the cut splanchnic nerves.

Further, handling of the intestine caused subsequent delay, presumably causing inhibitory splanchnic messages. To quote from their paper:

"Manipulations of the stomach and intestines, therefore, even gently and under the most favourable circumstances, produced in our experiments much greater effect in the direction of postoperative inactivity than any other of the factors under control during operation. Whether manipulation produces its effects on the mechanisms in the wall of the canal or indirectly through reflex inhibitions from the central nervous system was left undetermined."

\section{Dangers of Ileus.}

A slight distension of the intestines with gas is an almost constant occurrence after abdominal operations; it should begin to pass off in twelve to fifteen hours. Wher true ileus develops distension and discomfort steadily increase. The condition is a vicious circle, for the atony permits both stasis of faecal matter, which in putrefying causes further paralysis of the gut, and formation of gas, which increases the distension.

The dangers are readily apparent. (1) Organisms may penetrate the devitalized viscera and a fatal peritonitis occur. (2) Acute post-operative dilatation of the stomach may cause intractable vomiting and death from inanition and heart failure. (3) Kinking of the distended intestine may change the condition to mechanical intestinal obstruction. (4) Great distension may embarrass the diaphragm and impede the heart action.

More theoretical causes of death are: (a) Absorption via the lymphatics of a bacterial toxin found in the putrefying faecal matter (Murphy and Vincent ${ }^{3}$ ). (b) Splanchnic blood stasis, causing anaemia of the vital brain centres. (c) Irritation of the nerve endings in the intestinal wall, which causes death by constant reflex stimulation of the central nervous system. (d) The more recent work of Whipple and Stone ${ }^{4}$ suggests that the toxic symptoms are caused by the absorption by the blood stream of a toxin in the nature of a proteose which is secreted in the affected intestine.

Treatment of Ileus.

It cannot be denied that the present treatment of severe ileus is not all that could be desired; the condition is much feared by surgeons. Methods past and present are:

1. Massage of the abdomen. This is not enjoyed by the patient, nor is it ideal over a large wound.

2. Thrusting a trocar and cannula through the abdominal wall. This temporarily relieves one loop of bowel and infects the abdominal wall. It is not a scientific method.

3. Passage of a lighted taper or the cautery downwards along the colon, in the assumption that the heat would start peristalsis.

4. Temporary colostomy or enterostomy, hoping that if the gas and faecal contents escape peristalsis will be re-established. In practice, however, the loss of tone prohibits the emptying of the intestines.

5. For acute post-operative dilatation of the stomach washing out the stomach is certainly the best treatment. For atony of the intestines a stiff-walled rubber tube may be passed high up in the rectum and left in situ for several hours to permit the escape of flatus. High enemata of different kinds-soap, olive oil, turpentine and asafoetida, etc.-are of the utmost value.

6. Drugs acting from within. In ileus carminative and purgative drugs are disappointing.

7. Drugs acting from without.

(a) Physostigmine. For many years it has been known that physostigmine (eserine) has the power to augment the movements of the alimentary canal by stimulating the parasympathetic nerve ends. One of the first surgeons to use physostigmine as a remedy for post-operative ileus was Craig. ${ }^{5}$ Its value in this connexion was scientifically estimated by Cannon and Murphy, ${ }^{2}$ who found, however, that the contraction's 
it caused were short-lived. A few vears later Moennighoff ${ }^{7}$ even went so far as to recommend physostigmine in laparotomies as a prophylactic against distension, giving gr. 1/40 as a routine immediately the patient returned to bed. The other drug of creat value is-

(b) Pituitrin. The work of Dale ${ }^{8}$ proved scientifically the action of pituitrin: "The action of extracts of the posterior lobe of the pituitary body is a direct stimulation of involuntary muscle, without any relation to innervation. The action is most nearly allied to that of the digitalis series, but the effect on the heart is in this case slight, that on plain muscle intense." It was then clinically investigated by Bidwell. 9 This surgeon used pituitrin in a series of twenty-one cases of post-operative ileus. He found it of value, but concluded that it could not be completely relied on and should be used in conjunction with enemata.

The Action of Pituitrin and Physostigmine Combined.

At a meeting of the Association of Provincial Surgeons held at Oxford in 1914 Professor Gumn pointed out that in experiments on the intestines of laboratory animals there appeared to be a genuine synergism between pituitrin and physostigmine, evidenced by the fact that smaller doses of these substances in combination produced more pronounced stimulatory effect on the intestinal movements than could be produced by much larger doses of either of them singly; and he suggested that, in the treatment of post-operative ileus, a combination of pituitrin and physostigmine would probably be more efficacious than either alone. Since then at various times this combination has been tried at the Radcliffe Infirmary with good results, but no systematic attempt had been made to decide its value. Professor Gunn suggested to me that I should investigate this problem in the laboratory, using the technique employed by Gunn and Whitelocke for the isolated human appendix, and the present paper contains a summary of the results thus obtained as well as a record of a series of clinical cases in which the treatment was adopted during a period of six months. The large majority of cases of slight post-operative distension yielded to enemata. A certain number were more stubborn and required either pituitrin or physostigmine. The remainder were severe cases of ileus which did not improve with either drug separately. The combination was then used. The cases where the combination was used are as follows:

\section{CASE I.}

S. D., female, aged 62. Colostomy performed July 11th, 1922. The condition was general peritonitis, caused by a perforation through a growth in the region of the caecum; the whole of the mesentery and gut was covered with secondary deposits. Paul's tubes were tied in. Forty-eight hours later not even flatus had passed, so pituitrin 1/2 c.cm. was given twice during the following four hours; still nothing passed and the patient was practically moribund. Three hours later pituitrin $1 / 2 \mathrm{c.cm}$. with physostigmine gr. 1/100 was injected. A large motion resulted in half an hour. The patient improved (which was interesting scientifically, but regularly every day until she died on July 30th, 1922.

\section{CASE II.}

H. B., a woman aged 45, had felt ill for some hours, but on the morning of August 4th, 1922, performed her housework and did her morning shopping. Intense pain came on at 11 a.m. She was brought into hospital at noon and operated on at 5.30 p.m. Five feet of green-black gangrenous small intestine was found strangulated by an old adhesion. This was removed and a lateral anastomosis made. Physostigmine gr. 1/100 was given four-hourly from the time the patient left the theatre. In spite of this the patient had complete ileus till 11 o'clock the following evening, when pituitrin $1 / 2$ c.cm. with physostigmine gr. 1/100 was given. Twen pituitrin $1 / 2$ c.cm. with physostigmine gr. $1 / 100$ was given. Twenty minutes later she passed flatus and felt very much more
comfortable. She passed nothing further till 11 o'clock the following morning, when she was given pituitrin $1 \mathrm{c.cm}$. with physostigmine gr. 1/100. Twenty minutes later her bowels acted freely. She was regular afterwards and made an uninterrupted recovery.

\section{CASE III.}

M. G., female, aged 52. Hysterectomy performed on August 7th, 1922. This was followed by ileus of forty-six hours' duration. She was given physostigmine gr. 1/50, and in fifteen minutes passed a little fatus but had no alleviation of her pain. Three hours later pituitrin $1 / 2 \mathrm{c.cm}$. with physostigmine gr. $1 / 100$ was given. She had a good motion in twenty minutes and subsevered.

7. G.; male, aged 48. Appendix abscess operated on August post-operative dilatation of the stomach with very considerable vomiting, for which the stomach was washed out frequently. On the morning of August 11th, the fourth day after the operation,
the patient was greatly distended and had passed no trace of flatus. Pituitrin $1 / 2$ c.cm. was given with not the slightest result. At 6 p.m. pituitrin $1 / 2$ c.cm. with physostigmine gr. $1 / 100$ was injected. Within half an hour the patient had had three motions, was quite comfortable, and began a progressive recovery.

E. R., female, aged 5. Appendicectomy performed on August 8th, 1922. The appendix was found almost normal. On the following day much blood began to ooze from the wound and three stitches were put in. At the same time green projectile vomiting began. The next morning, two days after the operation, acute post-operative dilatation of the stomach was diagnosed. Pituitrin $1 / 2 \mathrm{cccm}$. was given at once, and two hours later the stomach was washed out and an enormous quantity of green vomit removed. After the anaesthetic (which was necessary because of the age of the patient) the child collapsed and had to have artificial respiration. Pituitrin 1/2 c.cm. was then given, and the patient came round slowly. Four hours later pituitrin $1 / 2 \mathrm{c.cm}$. with physostigmine gr. 1/200 was injected. Within half an hour she passed a small green motion; obviously some of the fluid in her stomach had passed right along the alimentary tract. There her stomach had passed right along the alimentary tract. There
was a similar very slight motion during the night. The next was a similar very slight motion during the night. The next morning the stomach was again washed out; no collapse followed;
nevertheless, pituitrin $1 / 2 \mathrm{c.cm}$. was given. All that day vomiting was incessant. In the evening the stomach was again washed out with smaller result, after which pituitrin $1 / 2 \mathrm{c.cm}$. with physostigmine gr. 1/100 was given. An hour later she passed a green began; the bowels opened regularly, she was on full diet two days later, and there was no more vomiting.

L. R., female, aged 50. Appendicectomy and salpingectomy performed August 25th, 1922. Ileus till morning of August 28th, when the patient was much distended and in great discomfort. At 11 a.m. pituitrin $1 / 2 \mathrm{c.cm}$. was given; absolutely nothing passed by the bowel and it only caused much belching and additional discomfort. At 3.30 p.m. pituitrin $1 / 2 \mathrm{c.cm}$. with physostigmine gr. 1/100 was injected, and ten minutes later the patient had a free motion and complete comfort after.

\section{CASE VII.}

F. B., female, aged 30 a primipara eight months pregnant. A parovarian cyst four inches in diameter prevented the head from engaging in the pelvis. Caesarean section was performed on as each day passed. Every method to open the bowels failed. After the first day physostigmine gr. $1 / 100$ was given sixhourly. On August 15th, four days after the operation, pituitrin $1 / 2$ c.cm. with physostigmine gr. 1/100 was given twice without any result. During this day vomiting had become intense; the any result. During this day romiting had become intense; the
stomach was washed out several times but there was no improrestomach was washed out several times but there was no improre-
ment. Per raginam and per rectum the pelvis seemed to be ment. Per raginam and per rectum the pelvis seemed to be few hours later. At the autopsy the uterus was found incar. cerated in the pelvis; the uterus must have been intensely contracted by the pituitrin, fallen back in the pelvis, then enlarged and in rising had become incarcerated, causing absolute mechanica] obstruction.

CASF VIrI.

K. C., female, aged 58. Hysterectomy performed August ع2nd, 1922. Ordered physostigmine gr. 1/100 four-hourly immediately after operation. Became increasingly distended during following three days and passed only slight amount of flatus. At 2 p.m. on the third day she had had about fifteen injections of physostigmine and there had been no improvement. Pituitrin $1 / 2 \mathrm{c.cm}$. with physostigmine gr. 1/100 was then given; one hour later she passed much flatus, and so on all the afternoon. Her bowels moved six hours after the injection, the distension passed off, and she was regular after.

A. P., male, aged 25. CASE IX. Three feet of ileum in front of the ileocaecal valve were distended, atonic, and injected. History of
old gunshot wound in abdomen, but no adhesions found. Next day patient was very distressed; intense vomiting and increasing distension. In the evening he was put on physostigmine gr. 1/100 four-hourly, alternating with strychnine gr. 1/60 four-hourly. The following day he was very much worse and the stomach was washed out several times. By 10.30 p.m. he had had four doses
of physostigmine and four doses of strychnine with no relief ; in fact he was worse. Pituitrin 1/2 c.cm. with physostigmine gr. 1/100 was given at 10.30 p.m.; during the next few hour's he passed much fatus and at $3.30 \mathrm{a} . \mathrm{m}$. he had a good motion. At $5.30 \mathrm{a} . \mathrm{m}$. he was given another dose of pituitrin $1 / 2 \mathrm{c.cm}$. with 5.30 a.m. he was given another dose of pituitrin $1 / 2$ c.cm. with
physostigmine gr. $1 / 100$. Shortly after he had two more good motions. An hour later the patient was an absolutely changed man, no longer in pain and wishing for food.

S. H., female, aged 38 . Hysterectomy performed September 15th, 1922. Increasing distension after operation; pain and loud complaints till 5.30 p.m. on September $18 \mathrm{th}$, when pituitrin $1 / 2 \mathrm{c.cm}$. with physostigmine gr. 1/100 was given. In twenty minutes the patient had a large motion followed by comiort and sleep.

M. W., female, aged 37. Operation on September 23rd, 1922, for the breaking down of adhesions from a previous operation. Physostigmine gr. 1/100 ordered four-hourly for the first day and six-hourly afterwards. By 6 p.m. on September 25th she was not much distended but very uncomfortable with "wind" which 
caused much belching. Pituitrin 1/2 c.cm. with physostigmine gr. 1/100 given. Twenty minutes later she passed a lot of flatus. An enerra or.e hour later resulted in a large motion.

\section{Case XIr.}

C. H., male, aged 41 . Operation on September 26th, 1922, for closure of a hernia through an old operation scar (appendix closure of a hernia through an old operation scar (appendix
abscess in 1894). On September 28th he was distended. Given abscess in 1894). On September 28th he was distended. Given
pituitrin $1 / 2 \mathrm{c.cm}$. with physostigmine gr. 1/100; twenty minutes pituitrin $1 / 2 \mathrm{c.cm}$. with physostigmine gr. $1 / 100$; twenty minutes
later he passed flatus. The next da.y acute post-operative dilatation of the stomach was diagnosed. Copious green vomit; stomach washed out and pituitrin and physostigmine given. Slight green stool half an hour later. On the two following days the patient was much better and began a little solid food. The stomach again had to be washed out on October 2nd as the vomiting suddenly returned-vomit yellow-brown; commencing heart failure. The next day he was worse, and he died on October 4th. At the autopsy the whole intestinal tract was found enormously dilated, the blood in the heart laked, and the heart muscle completely disorganized by a gas-forming organism. All his organs showed the ravages of an intense septicaemia. The wound had healed by first intention in spite of the strain of the vomiting.

Case xirr.

D. E., male, aged 37. Gangrenous appendix removed October 13th, 1922. Became increasingly distended till October 17th. The ligh rectal tube and enemata had only caused him to pass a very small amount of very small amount of 11.30 a.m. pituitrin $1 / 2 \mathrm{c.cm}$. with physostigmine gr. $1 / 100$ was injected. Five minutes later he had a large later he had a motion and passed puch flatus; the dition changed and he had a good night's sleep. In spite of a subsequent subphrenic abscess and empyema he made an excellent recovery.

Case xiv.

A. P., female, aged 44. Hysterectomy performed November 7th, 1922. By November 9th she had much distension and pain. distension and pain: with physostigmine with physostigmine
g r. $1 / 100$ given. Motion in ten minutes. Progressive recovery.

Case xv.

M. H., female, aged 44. Operation November 9th, 1922. Six feet of completely gangrenous small intestine herniated and strangulated through an opening in the mesentery. The patient was so ill before the operation that there was some doubt as to whether anything could be attempted. She improved slightly, however, as the operation went on, and finally complete resection of the gangrenous bowel and a lateral anastomosis was possible. Complete ileus developed, which was hardly surprising, although Complete ileus developed, which was hardly surprising, although
she was given physostigmine gr. $1 / 100$ six-hourly from the time she was given physostigmine gr. 1/100 six-hourly from the time
of the operation. By the morning of November 12th, three days later, the patient was in extrcmis-distended, vomiting, and in intense pain, hardly able to speak. Pituitrin 1/2 c.cm. with physostigmine gr. $1 / 100$ was then given. In ten minutes the patient had an enormous stool, nearly filling a large bed-pan. Half an hour later she began to improve markedly. That evening nutrient enemata were no longer needed as she was able to take a very little by mouth. The following evening she had a large natural motion, was quite hungry and able to commence a light diet.

Summary of Cases.

Cases II, IV, V, IX, and $\mathbf{X V}$ may be considered desperate cases and the relief given was dramatic. In Case II five feet and in Case XV six feet of gangrenous intestine was removed. In each case the risk of breaking down the anastomosis had to be taken, otherwise the patients would certainly have died. With regard to Cases VII and XII, the former had absolute mechanical obstruction from a uterus incarcerated in the pelvis; Cases xir was a genuine failure, for in spite of all efforts the patient succumbed to an intense general septicaemia.

EXPERIMENTAL Work.

Gunn and Underhill" found that " the isolated intestine still retains the power of executing rhythmical contractions, when warmed up in the usual way, after having been kept in Locke's solution at a temperature a few degrees above $0^{\circ}$ C. for three days." Later Gunn and Whitelocke ${ }^{11}$ investigated the movements of the isolated human appendix and the action of drugs upon it. I have employed the technique used by them. The appendices removed at the operation were dropped straight into a glass-stoppered bottle filled with Locke's solution and carried to the laboratory. The experiments were all carried out within one and a half hours of removal from the body. The appendices used in these experiments were all taken from young people. They were all very nearly normal, at the worst only slightly hyperaemic. Gunn and Whitelocke say:

"Our experiments have elicited the fact, which was not entirely to be expected, that a very severely inflamed appendix may still show spontaneous movements of a not definitely aberrant type. Indeed a piece of appendix immediately adjacent to a gangrenous portion which had ruptured through, showed no conspicuous impairment of movement."

In order to record the movements of the appendix the method used by Magnus ${ }^{12}$ for investigating movements of isolated mammalian intestine was employed. The appendix was suspended in a bath of Locke's solution, kept at a temperature of about $37^{\circ} \mathrm{C}$. The piece of appendix (about half an inch) was suspended between two hooks, the lower of which was fixed, while the upper, attached by a thread to a lever, recorded the contractions of the longitudinal muscle. The measured quantity of the drug was drawn into a pipette and blown into the beaker in which the appendix was suspended, the time of entry being marked on the tracing.

The accompanying tracings (Figs. 1 and 2) are extremely instructive. They are made by separate pieces of the same appendix, both experiments being carried out simultaneously on different drums. The interest is that in one case the pituitrin $1 / 2 \mathrm{c.cm}$. was given first and a slight rise obtained, then the physostigmine gr. $1 / 2400$, which caused a very much increased rise; while in the other the interval between the drugs was the same but the order reversed, the rise of tone being similar in each case. Neither drug alone produced much effect. Other experiments of this kind gave the same result.

\section{Summary and Conclusions.}

1. Experiments on the isolated human appendix have shown that physostigmine and pituitrin in combination have a more powerful effect in stimulating movements of the intestine than either of these singly.

2. A number of cases of severe post-operative atony are described in which this combination relieved the condition when physostigmine or pituitrin alone failed to do so. Indeed so far no case thus treated has failed unless where there was unsuspected mechanical obstruction.

I wish to thank Professor Gunn for help throughout, and Professor Heathcote for assistance with the laboratory work; also Mr. Whitelocke, Mr. Dodds-Parker, and Mr. Bevers, surgeons, and Mr. Hugh Whitelocke, assistant surgeon, to the Radcliffe Infirmary, for allowing me to publish records of patients under their care. The physostigmine used was the product of the British Drug Houses, Ltd.-gr. 1/100 tableis in hypodermic tubes. The pituitrin was the preparation of Burroughs, Wellcome and Co. Physostigmine (eserine) is a rather slowly soluble drug, so the tablet was 
usually dissolved in 5 minims of warm water and drawn into the syringe; the needle was then thrust into the pituitrin ampoule and its contents also drawn in and the combined solution injected subcutaneously.

REFERENCES TO LITERATURE.

1 Nothnagel: Diseases of the Intestine and Peritoneum, English translation, Philadelphia, 1904. 2 Cannon and Murphy, Journ. Amer. Med. As8oc., 49, 1907. 3 Murphy and Vincent B Boston Med. and Surg. journ., 65, 1911. "Whipple and Stone : Johns Hopkins Med. Bulletin. s Craig:! Amer. Journ. of Obst. and Dis. Women and Children, 49, 1904. B Craig : New York Med. Journ., March 13th, 1905. 7 Moennighoff: Journ. Missour State Mlel. As8oc., October, 1908. 8 Dale: Biochem. Journ., 4, 1909, p. 427. 9 Bidwcll: Journ. Amer. Med. A880c., 1911.10 Gunn and Underhill : Quart. Journ. Exper. Physiol., 8, 1914. 11 Gunn and Whitelocke : British
Journ. of Surgery, vol. 2, No. 5, 1914. 12 Magnus : Pflüger's Archiv, 1904.

.

\section{X-R.IY TREATMENT OF INTERSTITIAL KERATITIS.}

BY

\section{T. L. De COURCY, B.A., and J. H. MATHER, M.B.,} M.D.DuBL.,

HONORARY OPHTHALMIC SURGEON, Ch.B., D.M.R.E., ROYAL SOUTHERN HOSPITAL, LIVERPOOL.

THE value of treatment of various pathological conditions of the eye by $x$ rays has been recognized for some years, but the literature on the subject is scanty. In the earliest published cases a few unfavourable results were reported, probably owing to imperfect technique, but latterly some foreign writers have published cases showing marked benefit from $x$ rays carefully applied. We have come across few references to $x$-ray treatment of eye conditions in the British journals, and none as applied to interstitial keratitis.

Jendralski ${ }^{1}$ reports five cases of glioma of the retina successfully treated by $x$ rays and radium. In two of these a cure was claimed, whilst in the other three life was prolonged by a retardation of the rapidity of growth of the tumour cells. Other foreign writers detail cases of iridocyclitis, irido-choroiditis, uveitis, and vernal conjunctivitis treated by this method with good results, whilst Sgrosso, ${ }^{2}$ Japiot and Bussy, ${ }^{3}$ and Nicolle ${ }^{4}$ all report excellent results in cases of interstitial keratitis.

Parenchymatous or interstitial keratitis must be regarded as a disease of the uveal tract, of which the posterior layers of the cornea form a part, a disease also involving the iris and choroid. It used to be considered as essentially of syphilitic origin, regardless of the circumstances in which it took place. Sir Jonathan Hutchinson as recently as 1890 stated that it could not be the result of anything else. Atlhough the large majority of cases are of specific origin-generally inherited-there is no doubt that tuberculosis is also a very definite cause in a large number of cases, whilst malaria, trachoma, rickets, and focal infection (such as teeth, tonsils, sinusitis) have also proved to be the cause in isolated cases. A point recently emphasized by Butler ${ }^{5}$ is that trauma and operation are often factors precipitating the disease in a cornea predisposed to it by either syphilis or tuberculosis. Alyanakian $^{6}$ goes so far as to assert that interstitial keratitis is a dyscrasic affection, most frequently associated with hereditary syphilis, but that a determinant cause, such as acute infectious or chronic cachectic disease, is necessary in most cases for the disease to develop.

This all goes to show that the treatment of interstitial keratitis, arising as it does among persons whose powor of resistance is low, is often a very long affair beforo improvement takes place. It is still longer before the disease, having passed through the stages of infiltration and vascularization, reaches that of reabsorption.

Swanzy states the average duration of the acute stage to be six to eight weeks, or even longer, and we have all seen cases where the vascularizing stage has stretched into months, in spite of active treatment, leaving opacities so dense that vision has been reduced to hand movements. As the damage is done in the acute stage not only to the cornea but also to the whole uveal tract the chances of useful vision would be much improved if this stage could be cut short. Consequently, excessive infiltration and vascularization, together with the subsequent opacity, iritis, and choroiditis, might be minimized.
With this end in view we decided to try the effect of $x$ rays on certain selected cases at the Royal Souther'n Hospital, Liverpool, and we consider the results sufficiently striking to report. The number of cases treated is not large, but from the first application the effect of the $x$ rays was marked and improvement of the condition started almost immediately.

We have adopted the following technique, which is both simple and requires no special apparatus. The patient lies on his back on the couch, the head being firmly fixed by sandbags which are brought up level with the forehead on each side. A rectangular piece of thick felt about 14 inches by 6 inches, to which two layers of lead foil have been stitched, is taken, and in this are cut two circular windows approximately the size of the eyeballs and at the necessary distance apart. This pad is accurately centred, felt side down, over the eyes and held in position by means of additional sandbags. The tube stand, with a lead glass speculum just large enough to include the eyes, is placed in position so that the distance between the anticathode and the eye is 8 inches. A 7-inch gas tube is used, through which we pass a current of 1 milliampère backing up a 6-inch spark-gap, the rays being filtered through $0.5 \mathrm{~mm}$. of aluminium and one layer of felt. The duration of each treatment is six minutes. A treatment is given every seven days, and usually five or six suffice.

We have been led to adopt this " small dose" method with little filtration for the following reasons:

(a) The lesion treated is superficial, being mainly confined to the anterior chamber of the eye.

(b) We desire to give a stimulating or exciting dose. It is a well recognized fact that in such diseases as pruritus and certain forms of eczema small doses of soft rays are sufficient to effect rapid cures.

(c) With a small dose such as that described there is no risk of damage to the surrounding tissues; in no case have we caused redness of the skin or falling out of the eyelashes, and we have no reason to believe that any damage has occurred to the developing cells in the eyes of our young patients.

The following cases are illustrative of those treated:

Case I.

I. L., aged 13, presented a typical picture of severe interstitial keratitis affecting both eyes. Photophobia was present to keratitis affecting both eyes. Photophobia was present to
such a degree that the eyes could be opened only with difficulty, such a degree that the eyes could be opened only with difficulty,
lacrymation was profuse, and there was intense ciliary injection lacrymation was profuse, and there was intense ciliary injection
with vascularization of the cornea. Iritis was present and pain was with vascularization of the cornea. Iritis was present and pain was
exceedingly severe. The child had to have her bed shielded from the light and generally. was found kneeling under the bedclothes with her face buried in the pillow. The Wassermann reaction was positive, but tuberculous tests were not made. The case was singularly unresponsive to treatment although the usual remedies were applied-hot applications, atropine, dionine, calomel, together with intravenous injections of neo-kharsivan.

together with intravenous injections of neo-kharsivan.
Little change was apparent in spite of vigorous treatment till finally we decided to try $x$ rays. The applications were given weekly, and to us-it was the first acute case we had treated in this way - the effect was remarkable. After the first treatment photophobia and lacrymation were reliever; after the second the acute stage showed marked signs of abating, the ciliary injection had almost disappeared, and the child could sit up in bed happy and contented. After the third treatment she could read the large print of a child's book and was practically fit to leave hospital. The same treatment-local, $x$ rays, and specific-was, however, continued, and when last seen she was ready to go back however, continued, and when last seen she was ready to go back
to school, distance vision being-R. 6/18 partly, I. 6/12. Three were a few deposits on the lens capsule from posterior synechiae, and slight scarring of the cornea. There was no choroiditis. She had in all fourteen applications of $x$ rays, and apart from the relief experienced in the acute stage we consider that there was a decided beneficial effect on the opacities.

\section{Case Ir.}

D. A., aged 5, when first seen had severe interstitial keratitis of three weeks' duration in the right eye, photophobia and ciliary injection being very marked. The left eye showed early infiltration of the lower half of the cornea with ciliary injection. The Wassermann reaction was ++ , but it was decided not to submit the child to antisyphilitic treatment until the effects of $x$ rays the child to antisyphilitic treatment until the effects of $x$ rays had been observed. This treatment was commenced at once with
hot bathing and atropine locally. Eight days later the effect of the $x$-ray treatment was so marked that the acute stage was almost over. In this case there can be no doubt that $x$-ray treatment alone arrested the progress of the disease, the acute stage in the left eye having been cut short by several weeks. When last seen the opacity in each cornea was slight, and there is no doubt that no progression of infiltration had taken place since $x$ rays had been applied. 